\title{
Coexistencia de Síndrome Lifoproliferativo Crónico y Síndrome Mieloproliferativo Crónico en tres pacientes
}

\author{
M. C. HERNÁNDEZ SÁNCHEZ, H. GARCÍA QUIROGA , C. ULIBARRENA REDONDO, \\ J.A. MÉNDEZ SÁNCHEZ
}

Servicio de Hematología y Hemoterapia. Complejo Hospitalario de Ourense. ${ }^{I}$ Servicio de Hematología y Hemoterapia. Hospital Xeral-Calde. Lugo

CONCURRET LYMPHOPROLIFERATIVE AND MYELOPROLIFERATIVE DISORDERS IN THREE PATIENTS

\begin{abstract}
RESUMEN
Presentamos dos pacientes diagnosticados de Síndrome Linfoproliferativo Crónico: Leucemia linfocítica crónica B (LLC B) y LNH linfoplasmocítico, que desarrollaron un Síndrome Mieloproliferativo Crónico: Policitemia Vera (PV) y Leucemia Mieloide Crónica Philadelfia positivo (LMC) respectivamente y un tercer paciente con Síndrome Mieloproliferativo Crónico, Policitemia Vera, que desarrolló un S. Linfoproliferativo Crónico de inmunofenotipo "indefinido" y positividad para la ciclina D1. En la literatura hay pocos casos publicados y se desconoce si existe algún mecanismo común que explique la patogenia de ambos procesos y los mecanismos de control de un proceso sobre el otro.
\end{abstract}

PALABRAS CLAVE: Síndrome mieloproliferativo crónico. Síndrome linfoproliferativo crónico.

\begin{abstract}
We present two patients with a diagnosis of chronic lymphoproliferative syndrome, chronic lymphocytic leukemia $B(C L L B)$ and lymphoplasmacytic non-Hodgkin's lymphoma (NHL), who developed chronic myeloproliferative syndrome: polycythemia vera $(P V)$ and Philadelphiapositive chronic myeloid leukemia $(C M L)$ respectively, and a third patient with chronic myeloproliferative syndrome, polycythemia vera $(P V)$, who developed an undefined immunophenotype cycline $D^{I}$-positive chronic lymphoproliferative syndrome. The cases included in literature are scarce, and it is not known whether some common mechanism can explain both processes' pathogeneses and the control mechanisms of one process over the other.
\end{abstract}

KEY WORDS: Chronic myeloproliferative syndrome. Chronic lymphoproliferative syndrome.

Hernández Sánchez MC, García Quiroga H, Ulibarrena Redondo C, Méndez Sánchez JA. Coexistencia de Síndrome Linfoproliferativo Crónico y Síndrome Mieloproliferativo Crónico en tres pacientes. An Med Interna (Madrid) 2008; 25: 78-80.

\section{INTRODUCCIÓN}

La coexistencia de dos enfermedades hematológicas en un mismo paciente, una de estirpe mieloide y otra linfoide no es frecuente. Se han descrito casos, siempre escasos en número, en los que se diagnosticaron simultáneamente $(1,2)$ o bien, en el curso de uno de los procesos se diagnosticó el otro (3-8). En una búsqueda realizada en Embase y PubMed con los términos chronic lymphocitic leucemia, chronic lymphoproliferative syndrome, chronic myeloproliferative syndrome y sus combinaciones encontramos 19 publicaciones que presentaban 22 casos.

\section{CASOS APORTADOS}

-Caso1: varón de 93 años sin antecedentes de interés. En agosto de 1999 fue diagnosticado de LLC B, estadio 0/I de Rai, A de Binett, con inmunofenotipo típico, score 5 . No recibió tratamiento citorreductor y evolucionó como Smoldering LLC B, con linfocitosis menores a $20 \times 10^{9} / 1$. En mayo de 2003 presenta trombosis venosa profunda de MMII (TVP) y embolismo pulmonar (EP) y un $2^{\circ} \mathrm{EP}$ a los 3 meses de suspender la anticoagulación (TAO). En aquel momento los linfocitos eran 18 x 10\%/l, hemoglobina $(\mathrm{Hb}) 16 \mathrm{gr} / \mathrm{dl}$ y plaquetas 250 x 10\%/1. En febrero de 2004 se objetivó $\mathrm{Hb}$ de 18,6 gr/1, Hematocrito (Hcto) de $54 \%$ y, en el estudio de la masa eritrocitaria mediante ${ }^{51} \mathrm{Cr}$, un patrón de franca poliglobulia con vol. eritrocitario de $54,16 \mathrm{ml} / \mathrm{kg}$ (v.n: $<32 \mathrm{ml} / \mathrm{kg}$ ). Se descartaron causas secundarias de poliglobulia. No se realizó M.O por negativa del paciente. Cumplía criterios de Policitemia Vera (PVSG), aunque con niveles normales de eritropoyetina sérica y negatividad para la mutación V617F del Jak2 y BCR-XL y BCR-XS e incremento en la expresión de PRV1. Durante un año se realizaron sangrías terapéuticas (3-4 por trimestre) y no se indicó profilaxis con AAS porque el paciente se encontraba con TAO. En la actualidad, desde hace 4 meses, recibe hidroxiurea sin precisar, desde entonces nuevas sangrías. La cifra de leucocitos se mantiene entre $25-30$ x 10 $/ 1$, (linfocitos $60-80 \%$ ) y $\mathrm{Hb}$ alrededor de $14 \mathrm{gr} / \mathrm{dl}$

- Caso 2: varón de 59 años con artropatía psoriásica y FA que fue revertida a ritmo sinusal. En septiembre de 2003 por presentar ligero prurito y cefalea, se diagnosticó de Síndrome Mieloproliferati-

Trabajo aceptado: 17 de septiembre de 2008 
vo Crónico (SMPC) tipo Policitemia Vera (PV) con los criterios de la OMS, incluyendo la Eritropoyetina sérica disminuida, positividad para la mutación V617F del gen JAK2 y expresión aumentada del RNAm del PRV1. El gen BCL-XL y BCL- XS presenta niveles equivalentes. Se ha tratado con hidrea y recibe profilaxis con antiagregantes. A partir de febrero de 2006 se observó linfocitosis entre 5 y $10 \times 10^{9} / 1$ por lo que se procedió a estudio. En el aspirado medular existía una infiltración del $20 \%$ de linfocitos, de los cuales el $8 \%$ presentaba un inmunofenotipo similar al de los de S.P (CD 19++/5+/22++/23-/ 10-/ 11c-/25-/ 79b+/ 38-/FMC+-/bcl2+/ Ig SM $\lambda$ $+)$. El reordenamiento de BCR1/JH fue positivo para MCL3. La biopsia mostraba infiltración por S. Linfoproliferativo de bajo grado. La clínica, exploración y pruebas de imagen son normales. El paciente se ha diagnosticado de PV y Síndrome Linfoproliferativo Crónico de bajo grado BCL-1 positivo. Sigue en tratamiento con hidroxiurea y se mantiene vigilancia respecto al proceso linfoproliferativo.

- Caso 3: mujer de 62 años con antecedente de un herpes zoster y fracturas vertebrales dorsales y de arco costal atribuidas a osteoporosis. En estudio realizado por anemia sintomática y trombopenia, en nov de 1999, fue diagnosticada de Linfoma No Hodgkin (LNH) linfoplasmocítico. Recibió 8 c. COP seguidos de clorambucilo continuo hasta mayo de 2001 persistiendo en análisis posteriores una mínima banda monoclonal Ig $\mathrm{M} \lambda$ e infiltración medular residual por células CD 19+/5-/23-/22+/FCM7+/CD 25/11c-/ 79b+/SIgM $\lambda$, de inmunofenotipo similar al del diagnóstico. En mayo de 2003, con aumento de dolores óseos, se observa leucocitosis con precursores mieloides y basofilia, sugerente de SMPC. Se procede a estudio siendo diagnosticada de LMC, en fase crónica, bcr-abl +, p210, b3a2 y con $\mathrm{t}(9,22)$ (q34; q11) en el $100 \%$ de metafases. No se observó infiltrado linfoplasmocitario en el aspirado, biopsia ósea ni citometría. En la inmunofijación persistió un mínimo componente $\mathbf{M} \lambda$. Con Imatinib, $400 \mathrm{mg} / \mathrm{d}$, se ha conseguido una remisión molecular mantenida. En el control realizado en diciembre de 2006 se observa de nuevo una infiltración linfoplasmocítica en M.O (\%) que estamos vigilando.

\section{DISCUSIÓN}

El concepto que tenemos de las hemopatias mieloides que presentan nuestros pacientes es que se trata de enfermedades clonales, en las que se ha demostrado la implicación patogénica de genes mutados que tienen relación con la actividad tirosina kinasa. En el $60-90 \%$ de las PV la mutación puntual ocurre en el gen JAK2, situado en el cromosoma 9, en el cual la valina es sustituida por una fenilalanina en el codon 617. La expresión de la JAK2 V617F kinasa origina activación y crecimiento celular independiente de los factores de crecimiento y constituye un importante mecanismo patogénico en la PV, TE y MMA. En un amplio estudio realizado en 2005 por Levine y cols. (9) observa que la mutación JAK2 V617F ocurre en LMMC y LMA pero no en LLA o LLC indicando que está presente en procesos mieloides agudos y crónicos pero no en procesos linfoproliferativos. Por otro lado, no podemos olvidar que en las PV se ha descrito un mecanismo antiapoptótico que puede contribuir a que las células eritroides se acumulen. Una proteina que inhibe la apoptosis (Bcl-Xl) de la familia de las BCL2, se expresa de forma descontrolada e independiente de los factores de crecimiento. Por ello se considera la PV como una enfermedad mieloacumulativa (10). En el caso de la LMC el reordenamiento del gen bcr-abl, reflejo de la traslocación $\mathrm{t}$ $(9 ; 22)(q 34 ; q 11)$, da lugar a una proteina tirosinkinasa que ejerce una importante acción en los mecanismos de adhesión, apoptosis y proliferación celular.
En los procesos linfoproliferativos que nos ocupan en nuestros casos, también hay un origen clonal. En la LLC existe una proliferación clonal y acumulación de linfocitos B, aparentemente maduros, inmunocompetentes, con un inmunofenotípico característico. Las proteinas BCL-2 se hallan incrementadas habitualmente y disminuidas las del grupo BAX (favorecedoras de la apoptosis); eso se relaciona con los defectos de muerte celular programada de los linfocitos de la LLC y su acumulación en fase G0/G1(11). Un $n^{\circ}$ indeterminado de casos de $\mathrm{S}$ Linfoproliferativo Crónico con expresión periférica, permanecen mal definidos después de una cuidadosa evaluación inmunofenotípica y con frecuencia se denominan LLC atípica (12).

Con ayuda de la genética, se observa que algunos casos expresan la ciclina D1 (que es el resultado de una traslocación $\mathrm{t}(11 ; 14)$, lo que conlleva un acortamiento de G1 y aceleración de la proliferación celular) y pueden ser considerados linfomas del manto (13).

Nuestro paciente $n^{\circ} 2$ no presenta hasta el momento clínica, exploración o hallazgos que nos hagan pensar en un linfoma del manto, por lo que lo hemos catalogado como LLC atípica, si bien, estamos atentos a su evolución. Ignoramos si la presencia concomitante de la mutación V671F del JAK2 y la sobreexpresión de la ciclina D1 tienen alguna relación entre sí o son totalmente independientes. Los procesos tanto del paciente 1 como del 2 podrían tener en común algún cambio en los mecanismos de apoptosis que nosotros desconocemos y que produjera un carácter acumulativo en las células tanto mieloides como linfoides.

En la otra entidad clínica que padece uno de nuestros pacientes, el LNH linfoplasmocítico, se ha descrito, hasta en el $50 \%$, la traslocación $\mathrm{t}(9 ; 14)(\mathrm{p} 13 ; \mathrm{q} 32)$. Este reordenamiento involucra al gen PAX-5 en el cromosoma 9, un factor de trascripción específico de linfocitos $\mathrm{B}$, importante para la diferenciación de los precursores hacia la línea B, pero la única alteración cromosómica que por el momento hemos demostrado en la paciente es la que involucra al cromosoma 9 en el gen de fusión bcr-abl de la LMC.

Hay autores que especulan sobre la posibilidad de que los dos procesos ocurran como resultado de la expansión de una stem cell pluripotente $(5,14)$ sin llegarlo a demostrar. En general la mayoría $(1,4,6,15)$ consideran que la coexistencia de SMPC y SLPC, en un mismo paciente, es casual o existen dos proliferaciones independientes de dos líneas celulares diferentes, bajo un mismo estímulo antigénico. A veces, como en el caso de Ringbaek (3), la consideración de que es cuestión de azar se basa en la probabilidad estimada de que las dos condiciones ocurran independiente y simultáneamente teniendo en cuenta la incidencia anual de las dos entidades. Maher (16) llegó a demostrar el origen clonal independiente de una LLC y una LMC de un mismo paciente. Más recientemente, Hussein K y cols. (8) han demostrado estudiando el gen de la Janus Kinasa2 en un paciente con PV y LLC-B, que se trata de dos neoplasias que no comparten precursor común. Además, se ha visto que en SMPC, puede haber infiltrados linfocitarios clonales B, un 5\% (4 casos) de 81 en los que se pudo realizar estudio del reordenamiento del gen $\mathrm{Ig} \mathrm{H}$, sin que necesariamente tenga que existir un diagnóstico de linfoma (17) (en dos casos no había diagnostico de linfoma).

Es desconocido el efecto de control que una enfermedad puede ejercer sobre otra. Se ha descrito la supresión del clon leucémico de la LLC, cuando apareció el proceso mieloproliferativo(18) y viceversa, la remisión de la fase policitémica de 
PV después de la aparición de la LLC (19). De igual manera ocurre en algún caso en que la LLC se asoció a mielofibrosis (7). En nuestro caso $\mathrm{n}^{\circ} 3$ la población clonal residual del linfoma linfoplasmocítico desapareció cuando se diagnosticó de LMC. Sin embargo esa aparente remisión solo duró tres años sin que podamos explicar el por qué de estos acontecimientos. Desconocemos si de haber realizado bcr-abl cuando se diagnóstico el LNH linfoplasmocítico, éste hubiera sido positivo. En la actualidad hay remisión molecular de la LMC y no hay alteraciones en el cariotipo. Sólo hemos encontrado un caso de asociación entre S. Mieloproliferativo Crónico (PV) y linfoma linfoplasmocítico / macroglobulinemia de Waldenström que describe Saba (2). Se trata de un paciente que se diagnosticó simultáneamente de PV y Macroglobulinemia de Waldenström, en el que la ausencia de inactivación del cromosoma $X$ en células linfoides y no linfoides de S.P, y la ausencia reordenamientos en linfocitos $\mathrm{B}$ y $\mathrm{T}$ le lleva a conceder credi- bilidad a la idea de que existe una expansión clonal o no clonal dirigida por una o más citoquinas endógenas. Esto apoyaría la hipótesis de otros autores que defienden que un grupo de células malignas producirían una citoquina que a su vez causaría una segunda expansión clonal o no clonal.

La quimioterapia puede incrementar el riesgo de segundas neoplasias, sin embargo, no tiene fundamento achacar a los agentes terapéuticos utilizados en el paciente 2 y 3 implicación alguna en la aparición de los segundos procesos. En el caso 1 la PV se diagnosticó casi 5 años después de la LLC-B, sin que hubiera recibido tratamiento citostático previo.

En conclusión, creemos, que habrá que seguir investigando más casos en los que concurran a la vez enfermedad linfoproliferativa y mieloproliferativa, para, como apuntan estudios moleculares de otros autores, demostrar definitivamente si se trata de procesos que el azar hace coincidir en un mismo paciente y qué mecanismos de control ejercen unos procesos sobre otros.

\section{Bibliografía}

1. Hernández Nieto L, Raya Sánchez JM. Diagnóstico simultaneo de leucemia linfocítica crónica y Policitemia vera en un paciente. An Med Interna (Madrid) 2002; 19: 102-3.

2. Saba NF, Warth JA, Ross DG. Simultaneous occurrence of Policitemia vera and Waldenstrom macroglobulinemia: A case report and review of the literatura. Haematologica 2002; 32: 17-23.

3. Ringbaek Th and Schultz H. Coincidence of a Myeloproliferative and a lymphoproliferative Disorder- A Random Event? Leukemia 1988; 2: 472-3.

4. Fernández LLavador MJ, Ruiz Guinaldo MA, García Díaz M, et al. Síndrome linfoproliferativo crónico asociado a síndrome Mieloproliferativo. XLVIII Reunión Nacional AEHH y XXII Congreso Nacional SETH. Granada 26-28 octubre 2006. P-112.

5. Jacobsen N, Theilade K, Videbaek A. Tow additional cases of coexisting Polycythaemia Vera and Chronic Lymphocytic Leukaemia. Scand J Haematol 1982; 29: 405-10.

6. Hernández JJ, Appaiz I, Ramirez MM, et al. Paciente con coexistencia de un síndrome Mieloproliferativo crónico y un síndrome linfoproliferativo crónico. Haematologica 2004: 89 (Supl. 1): 354-6.

7. Hernández Nieto 1, Raya Sánchez JM, Álvarez Arguelles H, et al. A case of chronic lymphocytic leukemia overwhelmed by rapidly progressing idiopathic myelofibrosis. Haematologica 2000; 85: 973-7.

8. Hussein K, Brakensiek K, Ballmaier M, et al. B-CLL developing in a patient with PV is not affected by V617 mutation of the Janus Kinasa2. Eur J Haematol 2006; 77: 539-41.

9. Levine RL, Loriaux M, Huntly BJP, et al. The JAK2V617F activating mutation occurs in chronic myelomonocytic leukemia and acute myeloid leukemia, but not in acute lymphoblastic leukemia or chronic lymp-

hocytic leucemia. Blood 2005; 106: 3377-9.

10. Spivak JL. Policitemia vera: Myths, mechanisms and management. Blood 2002; 100: 4272-90.

11. Besses C, Sans Sabrafen. Síndromes Mieloproliferativos crónicos. Policitemia Vera y Trombocitemia Esencial. Sans-Sabrafen, ed. Hematología Clínica. $5^{\text {a }}$ edición. Elsevier. Madrid, 2006.

12. De Angeli C, Gandini D, Cuneo A, et al. BCL-1 rearrangements and p53 mutations in atypical chronic lymphocytic leukemia with $\mathrm{t}(11 ; 14)$ (q13;q32) Haematologica 2000; 85: 913-21.

13. Levy V; Ugo V; Delmer A et al. Cyclin D1 overexpression allows identification of an aggresive subset of elukemic lymphoproliferative disorders. Leukemia 1999; 13: 1343-51.

14. Januszewicz E, Firkin FC. Dual presentation of polycythaemia vera and lymphatic leucemia. Aust N Z J Med 1980; 10: 209-11

15. De Botelho de Sousa A, Gouveia J. Coexistent chronic lymphocytic leucemia and Policitemia vera requering no treatment. Med Oncol Tumor Phermacother 1989; 6: 239-40.

16. Maher VE, Gill L, Tornes PL et al. Simultaneous chronic lymphocitic leucemia and chronic myelogenous leucemia. Evidence of a separate stem cell origin. Cancer 1993; 71: 1993-7.

17. Pavor L, Lazca A, Kerestai L, et al. Increased incidente of monoclonal B-cell infiltrate in chronic myeloproliferative disorders. Modern Pathol 2004; 17: 1521-30.

18. Vianna NJ, Essman LJ. Suppression of chronic lymphocytic leukemia by polycythemia vera. Cancer 1971; 27: 1337-41.

19. Papayannis AG, Nikiforakis E, Asmagnostou-Keramida D. Development of chronic lymphocytic leucemia in a patient with polycythaemia vera. Scand J Haematol 1982; 29: 65-9. 